\title{
Realizing Strong Light-Matter Interactions between Single-Nanoparticle Plasmons and Molecular Excitons at Ambient Conditions
}

\author{
Gülis Zengin, ${ }^{1}$ Martin Wersäll, ${ }^{1}$ Sara Nilsson, ${ }^{1}$ Tomasz J. Antosiewicz,${ }^{1,2}$ Mikael Käll, ${ }^{1}$ and Timur Shegai ${ }^{1, *}$ \\ ${ }^{1}$ Department of Applied Physics, Chalmers University of Technology, 41296 Göteborg, Sweden \\ ${ }^{2}$ Centre of New Technologies, University of Warsaw, Banacha 2c, 02-097 Warszawa, Poland
}

(Received 5 December 2014; published 15 April 2015)

\begin{abstract}
Realizing strong light-matter interactions between individual two-level systems and resonating cavities in atomic and solid state systems opens up possibilities to study optical nonlinearities on a single-photon level, which can be useful for future quantum information processing networks. However, these efforts have been hampered by unfavorable experimental conditions, such as cryogenic temperatures and ultrahigh vacuum, required to study such systems and phenomena. Although several attempts to realize strong lightmatter interactions at room temperature using plasmon resonances have been made, successful realizations on the single-nanoparticle level are still lacking. Here, we demonstrate the strong coupling between plasmons confined within a single silver nanoprism and excitons in molecular $J$ aggregates at ambient conditions. Our findings show that deep subwavelength mode volumes $V$ together with quality factors $Q$ that are reasonably high for plasmonic nanostructures result in a strong-coupling figure of merit- $Q / \sqrt{V}$ as high as $\sim 6 \times 10^{3} \mu \mathrm{m}^{-3 / 2}$, a value comparable to state-of-the-art photonic crystal and microring resonator cavities. This suggests that plasmonic nanocavities, and specifically silver nanoprisms, can be used for room temperature quantum optics.
\end{abstract}

DOI: 10.1103/PhysRevLett.114.157401

PACS numbers: 78.67.Bf, 71.35.-y, 73.20.Mf, 78.66.Qn

Strong light-matter interactions are not only interesting from a fundamental quantum optics point of view, e.g., for studying entanglement and decoherence, but also because of their relevance for high-end emerging applications such as quantum cryptography [1], quantum networks [2], single-atom lasers [3], ultrafast single-photon switches [4], and quantum information processing [5-7]. These phenomena rely on a quantum emitter strongly interacting with a resonant cavity, which leads to cavity and emitter mode hybridization and vacuum Rabi splitting [8]. In the time domain, these strong light-matter interactions manifest themselves as a coherent exchange of energy between the cavity and the emitter occurring on time scales faster than both cavity and emitter dissipative dynamics-a situation that is dramatically different from irreversible spontaneous emission. Traditionally, these quantum optical phenomena have been studied in atomic $[9,10]$ and solid state systems [11-13], which are associated with considerable experimental challenges, such as ultrahigh vacuum, cryogenic temperatures, and fabrication issues.

A possible solution to these challenges could be to use noble metal nanoparticles instead of photonic crystal and microring resonator cavities [14-18]. This is because metal nanostructures can trap electromagnetic fields on subwavelength scales as so-called surface plasmon excitations. These plasmonic nanocavities possess a number of desirable properties, such as room temperature operation, deep subwavelength mode volumes, and nanoscale dimensions that have been shown to lead to many remarkable phenomena including single-molecule Raman spectroscopy [19-21], tip-enhanced imaging [22], ultracompact nanolasers [23], and enhanced fluorescence [24], to name a few. Despite these progresses, there has been considerably less success in achieving and demonstrating light-matter interactions in the strong-coupling regime in these structures.

Most experiments aiming at the realization of strong coupling utilize electronic excitations in a special kind of dye molecule aggregates, so-called $J$ aggregates, motivated by their exceptionally high oscillator strength and narrow resonances even at room temperature [25]. Recent studies along these lines include a variety of macroscopic or ensemble-type systems such as propagating surface plasmons in thin metal films, low- $Q$ Fabry-Perot resonators, and various nanoparticle arrays or assemblies coupled to a large number of excitons [26-31], as well as singlenanoparticle measurements [32-36]. However, to date, there remain ambiguities in the interpretation of the plasmon-exciton interactions in these structures [18], which from the quantum optics perspective translates into uncertainty in the number of excitons involved in the coupling process as well as into a question of whether plasmonic nanocavities are at all capable of realizing strong lightmatter interactions at its fundamental limit. These questions are crucial for potential quantum optics applications, since they require involvement of only a single exciton $[8,12,13]$.

Here we realized light-matter interaction in the strongcoupling regime between plasmons confined within single isolated silver nanoprisms and molecular excitons in $J$ aggregates at ambient conditions (Fig. 1). Our observations 


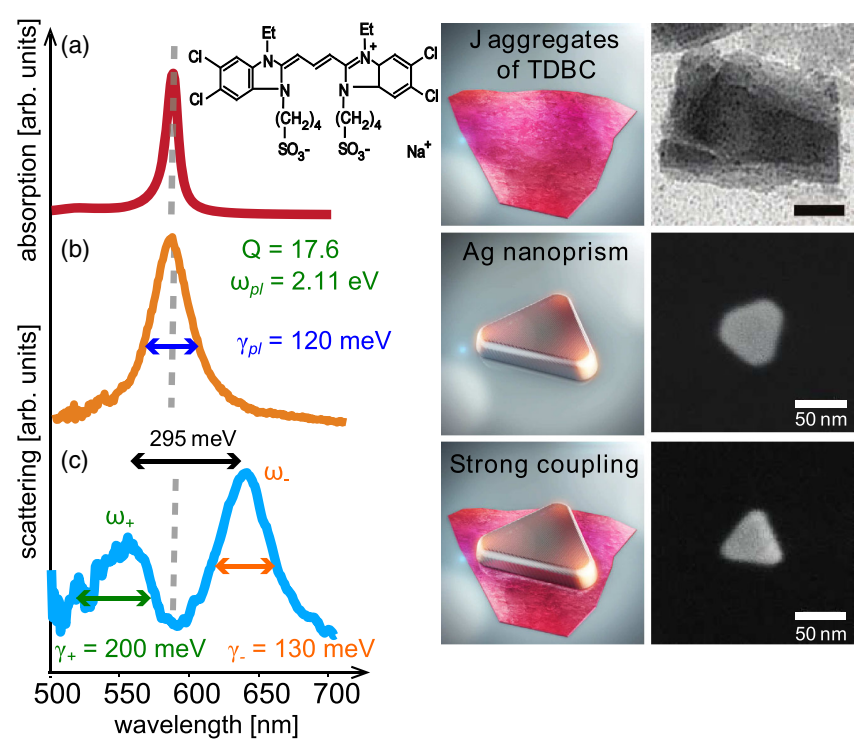

FIG. 1 (color online). Molecular excitons, nanoparticle plasmons, and coupling between them monitored by extinction or scattering spectroscopy and electron microscopy. (a) Left: Extinction spectrum of TDBC $J$ aggregates in water and chemical structure of TDBC monomer. Middle: Schematic view of a $J$ aggregate sheet. Right: Cryo-TEM image of several overlapped $J$-aggregate sheets. (b) Left: Scattering spectrum of a bare nanoprism with a quality factor of 17.6. Middle: Schematic view of a silver nanoprism. Right: SEM image of the prism. (c) Left: Scattering spectrum of single nanoprism strongly coupled to $J$ aggregates, resulting in a pronounced scattering dip. The hybrid plasmon-exciton branches, $\omega_{+}$and $\omega_{-}$, are split by $295 \mathrm{meV}$, while their full widths at half maxima are 200 and $130 \mathrm{meV}$, respectively. Middle: Schematic view of the coupled system. Right: SEM image of the corresponding prism. Scale bars are $50 \mathrm{~nm}$.

were facilitated by the weakly radiating nature of the silver nanoprisms and their small mode volumes $\left[V=(1-7) \times 10^{4} \mathrm{~nm}^{3}\right]$. We found that the plasmonexciton systems in our study exhibit $Q$ factors up to $\sim 20$, splitting-to-damping ratios $\left(2 g / \gamma_{\mathrm{pl}}\right)$ as high as $\sim 1.5$, and vacuum Rabi splitting up to $\sim 280 \mathrm{meV}$ involving $N \sim 70-85$ excitons in the mode volume. Furthermore, our morphological and spectral measurements and analysis of a wide range of samples reveal complex spectral features as a result of diversity of silver nanoprisms and inhomogeneous distribution of $J$ aggregates around the nanostructures, including almost $100 \%$ transparency dips and distinct Fano-shaped scattering spectra.

To achieve strong coupling between a cavity and an emitter, it is essential to combine a high- $Q-$ low- $V$ cavity with a high oscillator strength of the emitter. For this reason, we specifically chose silver nanoprisms and $J$ aggregates, since the former possess low damping due to small geometrical volumes and high crystalline quality, while the transition dipole moment $\mu_{J}$ of the latter is large due to a single electron excitation being delocalized over many adjacent molecules - all contributing to the oscillator strength [25]. The specific molecule we use is a cyanine dye called TDBC that forms $J$ aggregates in a water solution with a single exciton delocalized over $\sim 15$ molecules at room temperature [37]. Figure 1(a) shows the extinction spectrum of $J$ aggregates exhibiting a narrow peak at $588 \mathrm{~nm}\left(\omega_{0}=2.11 \mathrm{eV}\right)$. When a silver nanoprism supporting a plasmon resonance matching the $J$ band [Fig. 1(b)] is placed in close proximity to the molecules, a coupled hybrid system exhibiting very significant mode splitting into upper $\left(\omega_{+}\right)$and lower $\left(\omega_{-}\right)$hybrid plasmon-exciton branches is formed, signaling the realization of a strongcoupling scenario [Fig. 1(c)]. A possible dye-nanoparticle arrangement that could lead to such a scattering spectrum is schematically shown in Fig. 1-as supported by scanning and transmission electron (SEM and cryo-TEM) and atomic force microscopies showing that $J$ aggregates form thin $\sim 3 \mathrm{~nm}$ planar sheets and silver nanoprisms reside above or below them (see Fig. S1 in Supplemental Material [38]).

Figure 1 shows that silver nanoprisms can indeed strongly interact with $J$ aggregates. To reveal the factors affecting the coupling process, we measured and analyzed scattering spectra from $\sim 20$ isolated plasmon-exciton systems, structurally characterized them, and performed electrodynamics finite-difference time domain (FDTD) calculations. The individual silver nanoprisms' spectra were measured using hyperspectral imaging — an approach that allows for parallel sampling of many isolated particles [42,43] (Fig. S3 of Ref. [38]). Control experiments ensuring that the scattering dips indeed originate from plasmonexciton coupling were performed: monitoring spectral evolution as a function of $J$-aggregate photodecomposition (Fig. 3), fluorescence of hybrid systems (Fig. S8 of Ref. [38]), and bare silver nanoparticle scattering measurements (Figs. S4 and S9 of Ref. [38]).

To determine whether the plasmon-exciton system is strongly coupled, we describe our scattering spectra in terms of the classical coupled harmonic oscillator model, which predicts upper and lower plasmon-exciton branches in agreement with the quantum mechanical JaynesCummings picture [26,44,45]:

$$
\omega_{ \pm}=\frac{1}{2}\left(\omega_{\mathrm{pl}}+\omega_{0}\right) \pm \sqrt{g^{2}+\frac{\delta^{2}}{4}} .
$$

Here, $g$ is the coupling rate, $\omega_{\mathrm{pl}}$ and $\omega_{0}$ are plasmon and exciton resonance energies, and $\delta=\omega_{\mathrm{pl}}-\omega_{0}$ is the detuning. The plasmon-exciton branches can be directly accessed from the scattering data [Fig. 1(c)]. Assuming that the exciton resonance and width are homogeneous over the whole set of experiments $\left(\omega_{0} \approx 2.11 \mathrm{eV}\right.$ and $\left.\gamma_{0} \approx 100 \mathrm{meV}\right)$, we obtain the vacuum Rabi splitting $\Omega_{R}=2 g$, plasmon resonance, and plasmon linewidth from Eq. (1) as $\Omega_{R}=2 \sqrt{\left(\omega_{+}-\omega_{0}\right)\left(\omega_{0}-\omega_{-}\right)}$, 
$\omega_{\mathrm{pl}}=\omega_{+}+\omega_{-}-\omega_{0}$, and $\gamma_{\mathrm{pl}}=\gamma_{+}+\gamma_{-}-\gamma_{0}$, where $\gamma_{ \pm}$is the full width at half maximum of the corresponding plasmon-exciton branch. The quality factor is calculated as $Q=\left(\omega_{\mathrm{pl}} / \gamma_{\mathrm{pl}}\right)$. The coupled oscillator model is an alternative to direct determination of $\Omega_{R}$ via anticrossing at zero detuning, which is typically used in case of, e.g., tunable photonic crystal cavities [6]. Here the nanocavity resonances are not tunable; however, by looking at the upper and lower plasmon-exciton branches for all measured nanoprisms (Fig. S5 [38]), we observe that the resulting curves resemble an anticrossing behavior with $\Omega_{R}$ of $\sim 170 \mathrm{meV}$, in good agreement with Fig. 2(b). The analysis reveals that $\omega_{\mathrm{pl}}, \gamma_{\mathrm{pl}}, Q$, and $\Omega_{R}$ of the coupled plasmonexciton systems are distributed across a broad range of values [Figs. 2(b)-(e)], reflecting variations in particle morphology [Fig. 2(f)] as well as in the number of excitons contributing to the coupling process, $N$. Since $g=\sqrt{N} \mu_{J}\left|E_{\mathrm{vac}}\right|$, where $\left|E_{\mathrm{vac}}\right|=\sqrt{\hbar \omega / 2 \epsilon \epsilon_{0} V}$ is the vacuum field and $V$ is the mode volume [11], we should comment on the physical meaning of $V$ for plasmonic cavities. Following Refs. [46,47], we note that in case of small plasmonic nanoparticles the mode volume is well approximated by the geometrical volume, because the energy density $\epsilon|E|^{2}$ is concentrated mostly in the metal [Fig. 2(a) inset]. This implies that $V$ is given by the nanoprism side length $L$, as $V \sim L^{2}$, since the nanoprism's
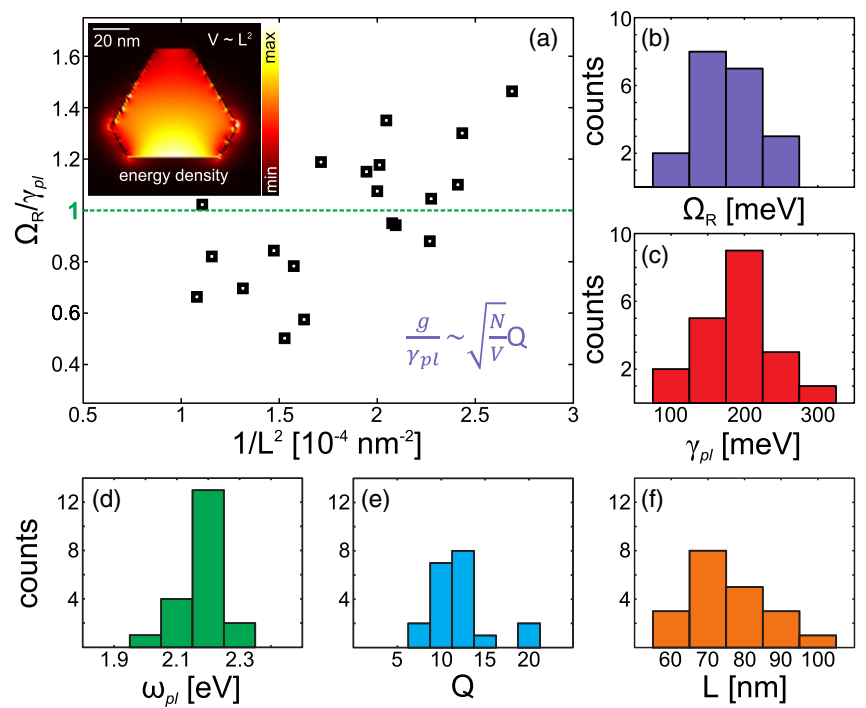

FIG. 2 (color online). Distribution of single-nanoparticle parameters extracted using the coupled oscillator model. (a) Ratio between Rabi splitting and plasmon damping $\left(2 g / \gamma_{\mathrm{pl}}\right)$ as a function of inverted geometrical volume $\left(L^{-2}\right)$. Strong coupling is reached when $2 g>\gamma_{\mathrm{pl}}$ is fulfilled. Inset: Energy density $\epsilon|E|^{2}$ distribution around a silver nanoprism, showing that $V$ is well approximated by geometrical volume, i.e., $V \sim L^{2}$. (b) Distributions of Rabi splitting $\Omega_{R}$, (c) plasmon damping $\gamma_{\mathrm{pl}}$, (d) plasmon resonance frequencies $\omega_{\mathrm{pl}}$, (e) quality factors, and (f) nanoprisms' side lengths. height is constant $10 \mathrm{~nm}$. Thus, the coupling process can be seen as being given by only two parameters: $N$ and $L$.

For further analysis we should first confine ourselves to a specific criterion of strong coupling taken as $2 g>\gamma_{\mathrm{pl}}$ in this study. This is a rather strict criterion in comparison to $2 g>\left(\gamma_{\mathrm{pl}}-\gamma_{0}\right) / 2$ or $2 g>\left(\gamma_{\mathrm{pl}}+\gamma_{0}\right) / 2$ used in other works $[6,35]$, and therefore satisfaction of this strict condition automatically implies satisfaction of all the weaker ones. The parameters extracted from the coupled oscillator model $-2 g, \omega_{\mathrm{pl}}$ and $\gamma_{\mathrm{pl}}$-allow us, on one hand, to infer whether the system satisfies the strong-coupling condition and, on the other hand, to estimate $N$ and $V$ through the standard relation for the coupling rate $g / \gamma_{\mathrm{pl}} \sim Q \sqrt{N / V}$ [11]. That said, in Fig. 2(a) we plot $2 g / \gamma_{\mathrm{pl}}$ as a function of $L^{-2}$ (mimicking dependence on $V$ ) and observe that several plasmon-exciton systems exhibit strong coupling, i.e., $2 g / \gamma_{\mathrm{pl}}>1$. We also observe that $2 g / \gamma_{\mathrm{pl}}$ increases for smaller particles; however, the correlation is not very strong, which is likely due to the diversity of the nanoprisms and inhomogeneous distribution of molecules around them. To verify whether such a spread is realistic, we performed electrodynamic FDTD calculations (Fig. S6 [38]), which show that silver nanoprisms indeed can be expected to have $Q$ values in the range of 8-14 and the mode volume in the range of $V=(1-7) \times 10^{4} \mathrm{~nm}^{3}$ in good correlation with geometrical volume. Such mode volume dispersion agrees well with the $2 g / \gamma_{\mathrm{pl}}$ spread shown in Fig. 2(a). We would like to stress here that a quantity $Q / \sqrt{V}$, which characterizes the cavity in terms of its coupling ability and is therefore referred to in the literature as the figure of merit for strong-coupling-related phenomena $[11,48]$, is about $\sim 6.3 \times 10^{3} \mu \mathrm{m}^{-3 / 2}$ in the case of the silver nanoprisms studied here $\left(Q=20\right.$ and $\left.V=10000 \mathrm{~nm}^{3}=10^{-5} \mu \mathrm{m}^{3}\right)$, which is only 5 times smaller than state-of-the-art photonic crystal cavities $\sim 3.2 \times 10^{4} \mu \mathrm{m}^{-3 / 2} \quad(Q=10000$ and $V=0.1 \mu \mathrm{m}^{3}$-parameters taken from Ref. [11]). This implies that plasmonic nanoparticles, especially in the form of the weakly radiating single-crystalline nanoprisms studied here, are indeed very prominent alternatives to photonic crystal and microring resonator cavities.

To elucidate how many excitons contribute to the observed interactions, we used the experimentally obtained values for $g$ together with numerically evaluated $V$, and compared them using the standard relations for the coupling rate and the vacuum field. For the two limiting cases representing the data $\left(g=140 \mathrm{meV}, V=10000 \mathrm{~nm}^{3}\right.$ and $\left.g=50 \mathrm{meV}, \quad V=70000 \mathrm{~nm}^{3}\right)$, we estimate $\sqrt{N} \mu_{J}=$ $\left(g /\left|E_{\mathrm{vac}}\right|\right) \approx 170-190$ D. $\mu_{J}$ is independently evaluated from the extinction measurement in a water solution of $J$ aggregates $\approx 20.5 \mathrm{D}$ (Fig. S2 [38]), and thus the overall number of excitons contributing to $g$ is $\approx 70-85$, while the number of TDBC monomers is $\sim 1000$. This number agrees well with alternative estimations based on the physical dimensions of hot areas around the nanoprisms (assuming $J$ 


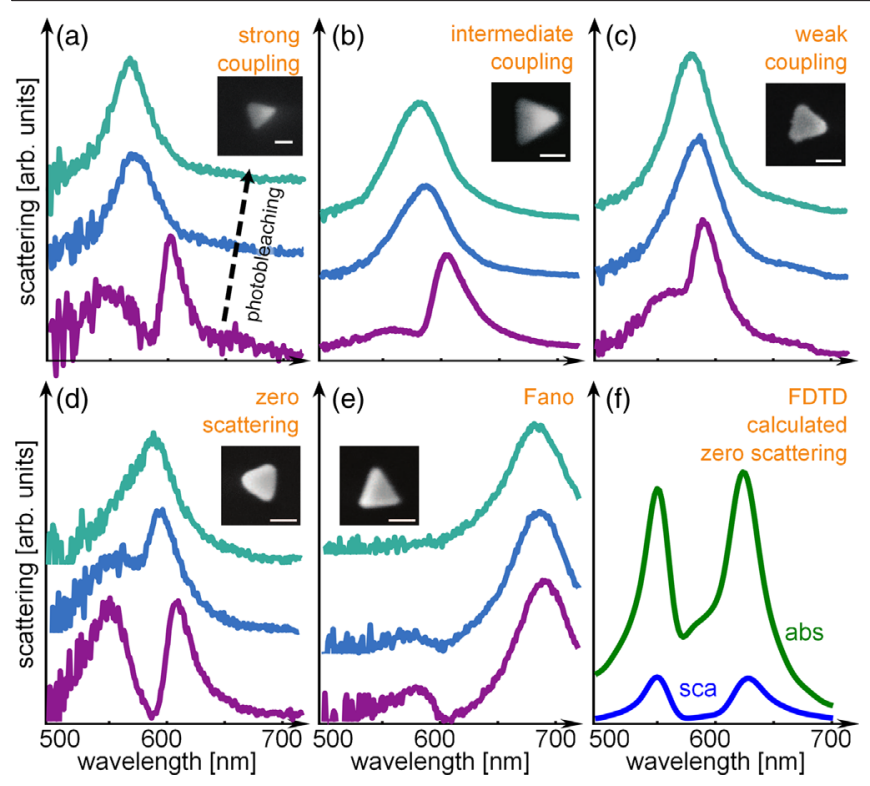

FIG. 3 (color online). Diversity of plasmon-exciton interactions. (a) A single-nanoparticle- $J$-aggregate system shown together with laser-induced $J$-aggregate photodegradation 2 and $10 \mathrm{~min}$ after continuous laser illumination for strongly coupled $2 g>\left(\gamma_{\mathrm{pl}}, \gamma_{0}\right)$, (b) intermediate coupling $\gamma_{\mathrm{pl}}>2 g>\gamma_{0}$, and (c) weak-coupling regimes $2 g \ll \gamma_{\mathrm{pl}}$. (d) Near-complete suppression of scattering (zero scattering) at around the $J$ band $(588 \mathrm{~nm})$ in the strong-coupling regime. (e) Fano-shaped scattering spectrum. SEM images of the nanoparticles are shown in insets. The scale bars are $50 \mathrm{~nm}$. (f) FDTD calculated absorption and scattering. Note the splitting of both cross sections.

aggregates are $3 \mathrm{~nm}$ thick and their molecular density is $\sim 1 \mathrm{~g} \mathrm{~cm}^{-3}$-typical for organic crystals like TDBC), as well as with FDTD calculations [38]. Although the current realization is rather far from the quantum optics limit, by extrapolating the coupling down to a single exciton we obtain $g=15 \mathrm{meV}$, which can be realistically increased by further compressing the mode volume and increasing $\mu_{J}$.

The plasmon-exciton system used in our study is amenable for realizing a wide range of light-matter interactions [Fig. 2(a)]. To illustrate this diversity we have chosen and characterized five of the nanoprisms in greater detail. The realized coupling regimes range from very strong to weak. Figure 3(a) shows a strongly coupled plasmon-exciton system with a Rabi splitting of $162 \mathrm{meV}$, while $\gamma_{\mathrm{pl}}=109 \mathrm{meV}$ and $Q \approx 20.2$, resulting in a value of $2 g / \gamma_{\mathrm{pl}}$ of about 1.5-a very high number for both plasmonic and photonic systems [6,35,36]. Importantly, Fig. 3(a) shows a system with a relatively modest Rabi splitting of $\sim 162 \mathrm{meV}$, while the maximum observed in this study is $\sim 280 \mathrm{meV}$, yet it is deep in the strongcoupling regime-achieved due to relatively high $Q$ in this case. In Fig. 3(b) we show the scattering spectrum for a nanoprism resembling a case of intermediate coupling $\left(\gamma_{\mathrm{pl}}>2 g>\gamma_{0}\right)$. Here, $2 g=151 \mathrm{meV}$, while $\gamma_{\mathrm{pl}}=184 \mathrm{meV}$ and $Q \approx 11.6$. The characteristic of this regime is that the dip is not as pronounced as in the strongcoupling case - a result of increased $\gamma_{\mathrm{pl}}$ and an average $Q$. However, coupling in the intermediate regime is still more pronounced than in the weak-coupling case shown in Fig. 3(c), where the scattering spectrum is only slightly suppressed at the position of the $J$-aggregate line-a situation realized when splitting is low, i.e., $\gamma_{\mathrm{pl}} \gg g$ $\left(\gamma_{\mathrm{pl}} \approx 191 \mathrm{meV}, \Omega_{R} \approx 96 \mathrm{meV}\right)$. Moreover, it has been shown previously that suppression of scattering in the case of weak coupling occurs mostly due to enhanced absorption in the dye layer [18]. A situation observed in Fig. 3(c) is similar to several previously published single-particle data [32-34], suggesting the realization of an enhancedabsorption scenario in these works.

Interestingly, the spectrum in Fig. 3(d) exhibits a nearly complete suppression of scattering in the strong-coupling regime (i.e., zero scattering), with $2 g \approx 200 \mathrm{meV}$ dominating over $\gamma_{\mathrm{pl}} \approx 174 \mathrm{meV}$. From Refs. [14,36], it can be shown that very pronounced dips in scattering response can be achieved when $\left(2 g / \sqrt{\gamma_{0} \gamma_{\mathrm{pl}}}\right) \gg 1$, which is true for Figs. 1(c), 3(a) and 3(d), suggesting that strong coupling is accompanied not only by large splitting but also by nearly complete suppression of scattering. The phenomenon of zero scattering requires that the absorption cross section of these nanostructures should also be strongly suppressed, in accordance with the optical theorem. To illustrate this, we perform FDTD calculations using realistic nanoprism- $J$-aggregate parameters [Fig. 3(f)], which indicate that spectral dips are present not only in scattering but also in absorption-as anticipated in the case of strong coupling [18].

Finally, in Fig. 3(e), we observe an asymmetric Fano line shape in the plasmon-exciton system, arising due to the interaction between a narrow $J$-aggregate resonance and a broad detuned plasmon resonance $\left(\omega_{\mathrm{pl}} \approx 1.83 \mathrm{eV} \ll \omega_{0}\right)$. This example aims at illustrating similarities between various aspects of plasmon-related phenomena often referred to as Fano resonances, strong coupling, or electromagnetically induced transparency.

In all five cases presented in Fig. 3, independently of the realized scenario, the scattering dips gradually disappeared upon laser-induced $J$-aggregate degradation, implying that the diversity arises directly from plasmon-exciton interactions. Indeed, SEM images confirm that in each case a spectrum originated from an isolated nanoprism. Fluorescence spectra of these plasmon-exciton systems (Fig. S8 [38]) are much broader than the fluorescence of the free $J$ aggregates in solution, indicating a strong interaction between $J$ aggregates and surface plasmons. Note that control experiments on bare silver nanoprisms showed that the laser illumination does not affect the nanoparticles' shape and spectra (Fig. S9 [38]).

In conclusion, strong light-matter interactions in the single-crystal nanoprisms studied here outperform all previously reported realizations, including $\mathrm{Ag}$ nanorods 
[36] and Au dimers [35], in terms of degree of coupling $2 g / \gamma_{\mathrm{pl}}$ and strong-coupling figure of merit $Q / \sqrt{V}$. We note that $2 g / \gamma_{\mathrm{pl}}$ and $Q / \sqrt{V}$ are more relevant for the description of strong coupling than the vacuum Rabi splitting alone, no matter how high the latter is. Indeed, ensemble measurements reported Rabi splitting approaching $\sim 1 \mathrm{eV}$ [49]— more than 3 times greater than in the current study; however, this was achieved due to a tremendous amount of molecules adsorbed within large mode volumes of macroscopic samples. Vacuum Rabi splitting in the range 200-400 meV was also claimed for the case of Au dimers produced by electron beam lithography [35]; however, these polycrystalline dimer structures exhibit significant radiative losses resulting in $\gamma_{\mathrm{pl}}$ as large as $\sim 370 \mathrm{meV}$, thereby reducing $2 g / \gamma_{\mathrm{pl}}$ to values of $\sim 1.08$ even for the only nanostructure displaying $\sim 400 \mathrm{meV}$ Rabi splitting. In the current study, $\gamma_{\mathrm{pl}}$ is strongly suppressed due to reduced radiative damping and the high crystalline quality of silver nanoprisms resulting in $2 g / \gamma_{\mathrm{pl}} \approx 1.5$ and $Q / \sqrt{V} \approx 6 \times 10^{3} \mu \mathrm{m}^{-3 / 2}$, as demonstrated by both experiments and calculations. These results allow us to estimate the number of excitons coherently contributing to the coupling process, $N \sim 70-85$, implying that singleexciton strong coupling might be within reach provided there is further compression of the mode volume. Furthermore, photobleaching and structural characterization experiments unambiguously prove that coupling arises due to plasmonexciton interactions. Importantly, our results show that plasmon-exciton systems are viable alternatives to photonic cavities, thereby opening exciting opportunities for room temperature quantum optics.

We would like to thank Dr. André Dankert for helping with AFM measurements, Annika Altskär for helping with cryo-TEM measurements, and Dr. Stefan Gustafsson for helping with TEM measurements. The authors acknowledge financial support from Swedish Research Council (VR), Knut and Alice Wallenberg Foundation (KAW), Swedish Foundation for Strategic Research (SSF), and the Foundation for Polish Science via the project HOMING PLUS/2013-7/1.

G. Z. and M. W. contributed equally to this work.

*timurs@chalmers.se

[1] H.-K. Lo and H. F. Chau, Science 283, 2050 (1999).

[2] T. G. Tiecke, J. D. Thompson, N. P. de Leon, L. R. Liu, V. Vuletić, and M. D. Lukin, Nature (London) 508, 241 (2014).

[3] J. McKeever, A. Boca, A. D. Boozer, J. R. Buck, and H. J. Kimble, Nature (London) 425, 268 (2003).

[4] T. Volz, A. Reinhard, M. Winger, A. Badolato, K. J. Hennessy, E. L. Hu, and A. Imamoglu, Nat. Photonics 6, 605 (2012).

[5] A. Imamoglu, D. D. Awschalom, G. Burkard, D. P. DiVincenzo, D. Loss, M. Sherwin, and A. Small, Phys. Rev. Lett. 83, 4204 (1999).
[6] G. Khitrova, M. Gibbs, M. Kira, S.W. Koch, and A. Scherer, Nat. Phys. 2, 81 (2006).

[7] D. E. Chang, V. Vuletic, and M. D. Lukin, Nat. Photonics 8, 685 (2014).

[8] K. Hennessy, A. Badolato, M. Winger, D. Gerace, M. Atatüre, S. Gulde, S. Fält, E. L. Hu, and A. Imamoglu, Nature (London) 445, 896 (2007).

[9] R. J. Thompson, G. Rempe, and H. J. Kimble, Phys. Rev. Lett. 68, 1132 (1992).

[10] T. Aoki, B. Dayan, E. Wilcut, W. P. Bowen, A. S. Parkins, T. J. Kippenberg, K. J. Vahala, and H. J. Kimble, Nature (London) 443, 671 (2006).

[11] T. Yoshie, A. Scherer, J. Hendrickson, G. Khitrova, H. M. Gibbs, G. Rupper, C. Ell, O. B. Shchekin, and D. G. Deppe, Nature (London) 432, 200 (2004).

[12] S. Gröblacher, T. Paterek, R. Kaltenbaek, C. Brunker, M. Zukowski, M. Aspelmeyer, and A. Zeilinger, Nature (London) 446, 871 (2007).

[13] A. Faraon, I. Fushman, D. Englund, N. Stoltz, P. Petroff, and J. Vucković, Nat. Phys. 4, 859 (2008).

[14] X. Wu, S. K. Gray, and M. Pelton, Opt. Express 18, 23633 (2010).

[15] S. Savasta, R. Saija, A. Ridolfo, O. Di Stefano, P. Denti, and F. Borghese, ACS Nano 4, 6369 (2010).

[16] A. Manjavacas, F. J. G. d. Abajo, and P. Nordlander, Nano Lett. 11, 2318 (2011).

[17] X.-W. Chen, V. Sandoghdar, and M. Agio, Phys. Rev. Lett. 110, 153605 (2013).

[18] T. J. Antosiewicz, S. P. Apell, and T. Shegai, ACS Photonics 1, 454 (2014).

[19] K. Kneipp, Y. Wang, H. Kneipp, L. T. Perelman, I. Itzkan, R. R. Dasari, and M.S. Feld, Phys. Rev. Lett. 78, 1667 (1997).

[20] S. Nie and S. R. Emory, Science 275, 1102 (1997).

[21] H. Xu, E. J. Bjerneld, M. Käll, and L. Börjesson, Phys. Rev. Lett. 83, 4357 (1999).

[22] R. Zhang, Y. Zhang, Z. C. Dong, S. Jiang, C. Zhang, L. G. Chen, L. Zhang, Y. Liao, J. Aizpurua, Y. Luo, J. L. Yang, and J. G. Hou, Nature (London) 498, 82 (2013).

[23] M. A. Noginov, G. Zhu, A. M. Belgrave, R. Bakker, V. M. Shalaev, E. E. Narimanov, S. Stout, E. Herz, T. Suteewong, and U. Wiesner, Nature (London) 460, 1110 (2009).

[24] A. Kinkhabwala, Z. Yu, S. Fan, Y. Avlasevich, K. Mülen, and W. E. Moerner, Nat. Photonics 3, 654 (2009).

[25] F. Würthner, T. E. Kaiser, and C. R. Saha-Möller, Angew. Chem., Int. Ed. Engl. 50, 3376 (2011).

[26] J. Bellessa, C. Bonnand, J. C. Plenet, and J. Mugnier, Phys. Rev. Lett. 93, 036404 (2004).

[27] J. Dintinger, S. Klein, F. Bustos, W. L. Barnes, and T. W. Ebbesen, Phys. Rev. B 71, 035424 (2005).

[28] P. Vasa, W. Wang, R. Pomraenke, M. Lammers, M. Maiuri, C. Manzoni, G. Cerullo, and C. Lienau, Nat. Photonics 7, 128 (2013).

[29] T. Schwartz, J. A. Hutchison, C. Genet, and T. W. Ebbesen, Phys. Rev. Lett. 106, 196405 (2011).

[30] S. Balci, Opt. Lett. 38, 4498 (2013).

[31] A. I. Väkeväinen, R. J. Moerland, H. T. Rekola, A.-P. Eskelinen, J.-P. Martikainen, D.-H. Kim, and P. Törmä, Nano Lett. 14, 1721 (2014).

[32] T. Uwada, R. Toyota, H. Masuhara, and T. Asahi, J. Phys. Chem. C 111, 1549 (2007). 
[33] G. L. Liu, Y. T. Long, Y. Choi, T. Kang, and L. P. Lee, Nat. Methods 4, 1015 (2007).

[34] W. Ni, T. Ambjönsson, S. P. Apell, H. Chen, and J. Wang, Nano Lett. 10, 77 (2010).

[35] A. E. Schlather, N. Large, A. S. Urban, P. Nordlander, and N. J. Halas, Nano Lett. 13, 3281 (2013).

[36] G. Zengin, G. Johansson, P. Johansson, T. J. Antosiewicz, M. Käll, and T. Shegai, Sci. Rep. 3, 3074 (2013).

[37] M. van Burgel, D. A. Wiersma, and K. Duppen, J. Chem. Phys. 102, 20 (1995).

[38] See Supplemental Material at http://link.aps.org/ supplemental/10.1103/PhysRevLett.114.157401 for detailed experimental methods, AFM measurements of $J$-aggregate-Ag-nanoparticle hybrids, numerical and experimental control experiments, Figs. S1-S9, and Refs. [39-41].

[39] R. Jin, Y. Cao, C. A. Mirkin, K. L. Kelly, G. C. Schatz, and J. G. Zheng, Science 294, 1901 (2001).
[40] R. C. Hilborn, Am. J. Phys. 50, 982 (1982).

[41] R. Ruppin, Phys. Lett. A 299, 309 (2002).

[42] J. M. Bingham, K. A. Willets, N. C. Shah, D. Q. Andrews, and R.P. Van Duyne, J. Phys. Chem. C 113, 16839 (2009).

[43] S. Chen, M. Svedendahl, R. P. van Duyne, and M. Käll, Nano Lett. 11, 1826 (2011).

[44] S. Rudin and T. L. Reinecke, Phys. Rev. B 59, 10227 (1999).

[45] V. M. Agranovich, M. Litinskaia, and D. G. Lidzey, Phys. Rev. B 67, 085311 (2003).

[46] A. F. Koenderink, Opt. Lett. 35, 4208 (2010).

[47] P. T. Kristensen and S. Hughes, ACS Photonics 1, 2 (2014).

[48] D. Englund, I. Fushman, and J. Vuckovic, Opt. Express 13, 5961 (2005).

[49] T. Schwartz, J. A. Hutchison, J. Léonard, C. Genet, S. Haacke, and T. W. Ebbesen, ChemPhysChem 14, 125 (2013). 